\section{Complicações pós-operatórias em tonsilectomias}

\author{
Vivian Wiikmann ${ }^{1}$, Flávio Augusto Passarelli \\ Prado $^{1}$, Marcello Caniello ${ }^{1}$, Renata C. Di \\ Francesco ${ }^{2}$, Ivan D. Miziara
}

\section{Post-operatory complications in adenotonsillectomy}

Resumo / Summary

\author{
Palavras-chave: tonsilectomia, complicações \\ cirúrgicas, hemorragia. \\ Key words: adenotonsillectomy, surgical \\ complications, bleeding.
}

\begin{abstract}
$\mathbf{A}$ denoidectomia e amigdalectomia são as cirurgias mais freqüentemente realizadas na prática otorrinolaringológica diária. Em geral, são procedimentos seguros, que não requerem internação prolongada. Em nosso serviço, o paciente recebe alta hospitalar cerca de seis horas após a intervenção, e são utilizadas diferentes técnicas cirúrgicas. Objetivo: Avaliar a segurança da liberação do paciente no mesmo dia e as complicações pós-operatórias, e compará-las às técnicas cirúrgicas utilizadas. Forma de estudo: Clínico prospectivo. Material e Método: Avaliamos prospectivamente 147 pacientes submetidos a adenoidectomia e/ou amigdalectomia por três técnicas diferentes no Hospital das Clínicas da FMUSP. Um protocolo foi aplicado pelo médico que realizou a cirurgia no pós-operatório imediato, após uma semana e um mês, pesquisando a presença de episódios de sangramento, febre, náuseas, vômitos, dor, disfagia a líquidos e a sólidos. Resultados: A incidência de hemorragia pós-operatória foi de 7,48\% no primeiro pós-operatório. Houve sangramento discreto em 3,4\% dos pacientes durante a primeira semana. Não houve diferença estatística entre os grupos em relação à técnica cirúrgica utilizada, quanto às complicações estudadas. Conclusão: A liberação do paciente após 6 horas de cirurgia é uma conduta segura. Como não há diferença estatística quanto às complicações de acordo com a técnica cirúrgica utilizada, acreditamos que o cirurgião deva utilizar a técnica com a qual é mais familiarizado.
\end{abstract}

\begin{abstract}
A denoidectomy and tonsillectomy are the most frequent surgeries been practice by otorhinolaryngologists. Usually adenotonsillectomy is a safe procedure, which does not request long hospitalization. In our service, patients are discharged from hospital about 6 hours after the end of the procedure. We regularly apply different surgical techniques for tonsillectomy. Aim: To evaluate the safeness of a day hospital system, and post-operatory complications, correlating them with the surgical techniques applied. Study design: Clinical prospective. Material and Method: We evaluated prospectively 147 patients submitted to tonsillectomies in our service. We applied three different techniques. The surgeon applied a protocol on the same day, a week and a month after procedure, inquiring about the occurrence of bleeding, fever, nausea, vomits, pain and inability to eat or drink. Results: The post-operatory incidence of bleeding was 7,48\% in the first day after procedure. Small amount of bleeding occurred in 3,4\% cases during the first week. No statistic difference was found correlating the surgical techniques applied and rated complications. Conclusions: Discharging patients from hospital 6 hours after surgery is a safe procedure. Considering that we found no statistic difference between the related complications and the surgical technique applied, we believe the surgeon must utilize his well-known technique.
\end{abstract}

\footnotetext{
${ }^{1}$ Médicos Residentes da Divisão de Clínica Otorrinolaringológica do Hospital das Clínicas da Faculdade de Medicina da Universidade de São Paulo.

${ }^{2}$ Médica Assistente do Hospital das Clínicas da Faculdade de Medicina da Universidade de São Paulo. Doutora em Otorrinolaringologia pela Faculdade de Medicina da Universidade de São Paulo.

${ }^{3}$ Professor Colaborador da Disciplina de Otorrinolaringologia do Hospital das Clínicas da Faculdade de Medicina da Universidade de São Paulo. Trabalho realizado na Divisão de Clínica Otorrinolaringológica do Hospital das Clínicas da Faculdade de Medicina da Universidade de São Paulo. Endereço para correspondência: Dr. Marcello Caniello - Rua Dr. Ovídio Pires de Campos, 171/206 São Paulo SP 05403-010 Tel (0xx11) 3082-5482/9693-4705 - E- mail: mcaniello@ig.com.br

Artigo recebido em 02 de abril de 2004. Artigo aceito em 17 de junho de 2004
} 


\section{INTRODUÇÃO}

Adenoidectomia e amigdalectomia são as cirurgias mais freqüentemente realizadas no Departamento de Otorrinolaringologia do Hospital das Clínicas da Faculdade de Medicina da Universidade de São Paulo e na prática otorrinolaringológica diária ${ }^{1,2,3}$. Em geral, são procedimentos seguros, que não requerem internação prolongada. Em nosso serviço, os pacientes submetidos a esta cirurgia, se não apresentam complicações, recebem alta hospitalar no mesmo dia e são acompanhados ambulatorialmente após uma semana e um mês de pós-operatório. Mas esta não é a regra em outros serviços, em que os pacientes chegam a ficar internados até no mínimo cinco dias ${ }^{3}$.

As principais técnicas cirúrgicas utilizadas em nosso serviço são: A técnica de Sluder-Ballenger, introduzida por Sluder em 1911, que inclui preensão das tonsilas com pinça específica e dissecção digital do seu plano subcapsular, sob anestesia geral, dissecção com pinça Hartmann, onde o plano subcapsular é dissecado com essa pinça e dissecção com aspirador-descolador, instrumento com uma extremidade em forma de foice serrilhada, que pode dissecar o plano subcapsular e aspirar o sangramento excedente simultaneamente ${ }^{4}$.

Apesar de ser um procedimento seguro, as complicações pós-operatórias, quando ocorrem, podem oferecer risco de vida ao paciente, como no caso de hemorragia pósoperatória. Esta última tem incidência de 10\%3e é considerada a complicação mais grave. Podemos classificá-la como sendo primária (<24 horas) ou secundária ( $>24$ horas) ${ }^{3}$. A maioria dos estudos atesta que a hemorragia primária é muito mais freqüente que a secundária ${ }^{5,6}$, sendo que este tipo de evento ocorre entre 6 e 8 horas de pós-operatório na maioria das vezes $(76-89 \% \text { dos casos })^{2}$.

Outras complicações pós-operatórias comuns são náuseas, vômitos, febre, inabilidade para ingerir alimentos sólidos ou líquidos, otalgia e dor ${ }^{2,7}$. Lee \& Sharp ${ }^{7}$ observaram necessidade de analgesia em 92,4\% e náuseas em 20,3\% dos pacientes no primeiro pós-operatório. No mesmo período, Rivas Lacarte ${ }^{8}$ relatou febre em 5,52\% dos casos. A incidência de vômitos varia de $1,57 \%$ a 33\% $0^{7,9}$.

Algumas destas complicações podem atingir grande magnitude, como por exemplo, as que determinam desidratação e necessidade de reposição volêmica.

\section{OBJETIVOS}

Tendo em vista que em nosso serviço rotineiramente o paciente recebe alta hospitalar cerca de seis horas após a intervenção cirúrgica, período em que ocorre a grande maioria dos casos de hemorragia pós-operatória ${ }^{2}$, o presente estudo tem por objetivo avaliar as conseqüências e a segurança desta conduta, bem como as principais complicações pós-operatórias descritas na literatura. Avaliamos a ocor- rência de sangramento pós-operatório, náuseas, vômitos, febre, dor e disfagia, sua freqüência e magnitude, e o período de maior incidência destes.

Outro de nossos objetivos foi a comparação da incidência de complicações pós-operatórias de acordo com a técnica cirúrgica utilizada (técnica de Sluder-Ballenger, dissecção com pinça de Hartmann ou descolador-aspirador).

\section{MATERIAL E MÉTODO}

Avaliou-se prospectivamente 147 pacientes submetidos a tonsilectomia no período de março a dezembro de 2002 no Departamento de Otorrinolaringologia do Hospital das Clínicas da Faculdade de Medicina da Universidade de São Paulo. Os critérios de indicação cirúrgica utilizados foram de acordo com o Consenso da Sociedade Brasileira de Otorrinolaringologia. ${ }^{10}$

Os pacientes foram selecionados para cirurgia baseado em anamnese, exame físico e exames complementares, como Raios-X de cavum, fibroscopia e audiometria, quando indicados. Foi feita avaliação pré-operatória laboratorial em todos os pacientes, utilizando hemograma completo, tempo de trombina, tempo de protrombina e tempo de tromboplastina. Se houve necessidade, os pacientes foram avaliados por médico hematologista previamente à cirurgia, sendo que foram excluídos os que apresentavam doenças hematológicas.

Todos os pacientes foram submetidos a cirurgia sob anestesia geral e intubação orotraqueal. Amigdalectomia foi realizada através das técnicas de Sluder-Ballenger (6 pacientes), dissecção com pinça de Hartmann (43 pacientes) ou com uso do descolador-aspirador de amígdalas (80 pacientes). A adenoidectomia foi realizada por meio de sua curetagem, com a cureta de Lermoyez.

Os pacientes que não apresentaram complicações após seis horas de cirurgia receberam alta hospitalar para seguimento ambulatorial. O acompanhamento foi realizado em uma semana e um mês de pós-operatório. Novos retornos foram marcados em casos de complicações que necessitaram de seguimento mais freqüente.

Foram avaliadas as principais complicações descritas na literatura referentes a pós-operatório de tonsilectomias imediatamente após a cirurgia, após uma semana e em um mês.

Protocolo foi aplicado pelo médico que realizou a cirurgia no pós-operatório imediato, e em seguimento ambulatorial após uma semana e um mês, pesquisando dados demográficos, bem como a presença de episódios de sangramento, febre (temperatura axilar acima de $38^{\circ} \mathrm{C}$ ), náuseas, vômitos, dor, disfagia a líquidos e a sólidos. Anotamos também o tipo de técnica cirúrgica aplicada a cada caso. Consideramos em nosso estudo hemorragias em qualquer intensidade, a fim de quantificá-las em termos de repercussão clínica para o paciente. 
Todos os pacientes ou seus responsáveis assinaram termo de consentimento informando a respeito dos riscos e possíveis complicações do ato cirúrgico, e este protocolo foi aprovado pelo comitê de ética do Hospital das Clínicas da Faculdade de Medicina da Universidade de São Paulo.

Os pacientes foram divididos inicialmente em três grupos: os submetidos a tonsilectomia (A1), os submetidos a adenotonsilectomia (A2) e os submetidos a adenoidectomia (Ad).

Os pacientes submetidos a amigdalectomia foram divididos segundo a técnica intra-operatória utilizada: dissecção com pinça Hartmann, utilização de aspirador descolador e técnica de Sluder.

A análise estatística em relação as complicações foi realizada utilizando-se o teste Qui-quadrado ${ }^{11}$ ou o teste exato de Fisher ${ }^{11}$.

A comparação dos grupos em relação a variável idade foi realizada através da Análise de Variância a um fator ${ }^{11}$, com comparações múltiplas através do teste de Bonferroni ${ }^{11}$. O nível de significância utilizado para os testes foi de 5\%.

\section{RESULTADOS}

Foram avaliados 147 pacientes com faixa etária entre 2 e 47 anos, com média de 8,9 anos, sendo $42,2 \%$ do sexo feminino e $57,8 \%$ do sexo masculino.

Os resultados quanto à sintomatologia nos três períodos avaliados, bem como a comparação estatística entre eles, estão listados nas Tabelas 1 e 2.

Utilizando-se teste qui-quadrado, nota-se que os resultados obtidos no primeiro pós-operatório, com uma semana e um mês de pós-operatório são estatisticamente diferentes.

No $1^{\circ}$ pós-operatório, temos diferença significante entre os grupos com relação à disfagia a líquidos, apresentada de forma importante pelos pacientes submetidos a amigdalectomia (A1). Com uma semana de pós-operatório obtivemos diferença significante entre os grupos em relação a dor e disfagia a sólidos, que foi mais freqüente no grupo submetido a A1 e com um mês de pós-operatório temos diferença significante entre os grupos em relação a dor, também mais freqüente no grupo A1.

Tabela 1. Comparação entre os sintomas e os grupos (em números absolutos).

\begin{tabular}{|c|c|c|c|c|c|c|c|c|c|c|c|c|}
\hline \multirow{2}{*}{$\begin{array}{l}\text { Pós-operatório } \\
\text { Sintomas }\end{array}$} & \multicolumn{4}{|c|}{$1^{\circ} \mathrm{PO}$} & \multicolumn{4}{|c|}{$7^{\circ} \mathrm{PO}$} & \multicolumn{4}{|c|}{$30^{\circ} \mathrm{PO}$} \\
\hline & $\begin{array}{c}A 1 \\
(n=18) \star\end{array}$ & $\begin{array}{c}\text { A2 } \\
(n=101)^{*}\end{array}$ & $\begin{array}{c}\text { Ad } \\
(n=28)^{\star}\end{array}$ & $\mathrm{p}$ & $\begin{array}{c}\text { A1 } \\
(n=18)\end{array}$ & $\begin{array}{c}A 2 \\
(n=101)\end{array}$ & $\begin{array}{c}\text { Ad } \\
(\mathrm{N}=28)\end{array}$ & $\mathrm{p}$ & $\begin{array}{c}\mathrm{A} 1 \\
(\mathrm{~N}=18)\end{array}$ & $\begin{array}{c}A 2 \\
(N=101)\end{array}$ & $\begin{array}{c}\text { Ad } \\
(N=28)\end{array}$ & $p$ \\
\hline Nauseas & 5 & 26 & 2 & 0,096 & 0 & 9 & 1 & 0,456 & 0 & 1 & 0 & 1,000 \\
\hline Vômitos & 2 & 23 & 3 & 0,234 & 0 & 10 & 0 & 0,106 & 0 & 1 & 0 & 1,000 \\
\hline Febre & 0 & 8 & 2 & 0,766 & 0 & 13 & 1 & 0,161 & 0 & 3 & 0 & 1,000 \\
\hline Otalgia & 0 & 10 & 1 & 0,375 & 3 & 18 & 3 & 0,739 & 0 & 3 & 1 & 1,000 \\
\hline Disfagia a líquidos & 8 & 14 & 4 & 0,013 & 1 & 4 & 0 & 0,480 & 0 & 0 & 0 & 0 \\
\hline Disfagia a sólidos & 11 & 51 & 6 & 0,425 & 10 & 27 & 0 & 0,001 & 0 & 1 & 0 & 1,000 \\
\hline Dor & 13 & 64 & 15 & 0,290 & 12 & 42 & 3 & 0,001 & 2 & 0 & 0 & 0,014 \\
\hline Sangramento & 3 & 7 & 1 & 0,266 & 1 & 4 & 0 & 0,480 & 0 & 0 & 0 & 0 \\
\hline Reoperação & 1 & 1 & 0 & 0 & 0 & 0 & 0 & 0 & 0 & 0 & 0 & 0 \\
\hline
\end{tabular}

$\mathrm{N}=$ número total de casos. $\mathrm{p}=$ nível de significância

* $\mathrm{A} 1$ = amigdalectomia, $\mathrm{A} 2$ = tonsilectomia, $\mathrm{Ad}=$ adenoidectomia

Tabela 2. Comparação entre os sintomas e as técnicas utilizadas (em porcentagem).

\begin{tabular}{|c|c|c|c|c|c|c|c|c|c|c|c|c|}
\hline \multirow{2}{*}{$\begin{array}{l}\text { Pós-operatório } \\
\text { Sintomas }\end{array}$} & \multicolumn{4}{|c|}{$1^{\circ} \mathrm{PO}$} & \multicolumn{4}{|c|}{$7^{\circ} \mathrm{PO}$} & \multicolumn{4}{|c|}{$30^{\circ} \mathrm{PO}$} \\
\hline & $\begin{array}{c}\text { Asp* } \\
(\mathrm{N}=80)\end{array}$ & $\begin{array}{l}\text { Hart* }^{*} \\
(\mathrm{~N}=43)\end{array}$ & $\begin{array}{l}\text { Slud* } \\
(\mathrm{N}=6)\end{array}$ & $p$ & $\begin{array}{c}\text { Asp } \\
(\mathrm{N}=80)\end{array}$ & $\begin{array}{c}\text { Hart } \\
(\mathrm{N}=43)\end{array}$ & $\begin{array}{l}\text { Slud } \\
(\mathrm{N}=6)\end{array}$ & $p$ & $\begin{array}{c}\text { Asp } \\
(\mathrm{N}=80)\end{array}$ & $\begin{array}{c}\text { Hart } \\
(\mathrm{N}=43)\end{array}$ & $\begin{array}{l}\text { Slud } \\
(\mathrm{N}=6)\end{array}$ & $p$ \\
\hline Nauseas & 29,03 & 33,33 & 66,67 & 0,390 & 6,45 & 15,38 & 33,33 & 0,167 & 0 & 2,56 & 0 & 1,000 \\
\hline Vômitos & 29,03 & 23,08 & 66,67 & 0,230 & 6,45 & 15,38 & 0 & 0,499 & 0 & 2,56 & 0 & 1,000 \\
\hline Febre & 9,68 & 10,26 & 0 & 1,000 & 19,35 & 10,26 & 0 & 0,566 & 3,23 & 5,13 & 0 & 1,000 \\
\hline Otalgia & 19,35 & 10,26 & 0 & 0,566 & 19,35 & 20,51 & 33,33 & 0,781 & $6,, 45$ & 2,56 & 0 & 0,631 \\
\hline Disfagia a líquidos & 12,9 & 17,95 & 0 & 0,844 & 0 & 5,13 & 0 & 0,540 & 0 & 0 & 0 & 0 \\
\hline Disfagia a sólidos & 38,71 & 82,55 & 33,3 & & 29,03 & 33,33 & 66,67 & 0,390 & 0 & 2,56 & 0 & 1,000 \\
\hline Dor & 74,19 & 82,05 & 66,67 & 0,533 & 38,71 & 53,85 & 66,67 & 0,417 & 0 & 0 & 0 & - \\
\hline Sangramento & 3,23 & 7,69 & 0 & 0,683 & 6,45 & 5,13 & 0 & 1,000 & 0 & 0 & 0 & - \\
\hline Reoperação & 0 & 0 & 0 & 0 & 0 & 0 & 0 & 0 & 0 & 0 & 0 & - \\
\hline
\end{tabular}

Asp = técnica utilizando aspirador-descolador

Hart = técnica utilizando pinça de Hartmann

Slud = técnica utilizando guilhotina de Sluder 
Com relação à hemorragia, observamos maior incidência de sangramento no primeiro pós-operatório em relação à primeira semana e primeiro mês. Não houve diferença estatisticamente significante entre os grupos com relação a náuseas, vômitos, febre e otalgia no primeiro pós-operatório, em uma semana e um mês. Em dois casos houve necessidade de reabordagem cirúrgica devido a sangramento, nos grupos A1 e A2.

Finalmente, o grupo de pacientes submetidos a tonsilectomia foi dividido segundo a técnica intra-operatória utilizada: dissecção com pinça Hartmann (43 pacientes), utilização de aspirador descolador (80 pacientes) e utilização de técnica de Sluder (6 pacientes) (Tabela 2).

Observamos que não houve diferença significante entre as técnicas utilizadas para a realização da amigdalectomia, com relação às complicações estudadas.

\section{DISCUSSÃO}

O alto custo dos procedimentos médicos e hospitalares é assunto de suma importância na medicina moderna. Em alguns centros ainda é pratica habitual manter os pacientes submetidos a tonsilectomias internados por vários dias após a cirurgia. Observamos com relação à incidência de hemorragia pós-operatória, que 11 pacientes apresentaram sangramento no primeiro pós-operatório $(7,48 \%)$, sendo que dois destes apresentaram sangramento importante, tendo sido submetidos à revisão cirúrgica (1,36\%) durante as primeiras 6 horas de pós-operatório. Estes resultados foram comparáveis ao de outros serviços, em outros países. ${ }^{3,4,5}$

Durante a primeira semana após a cirurgia, 5 pacientes $(3,4 \%)$ apresentaram episódios de sangramento, que foram leves e melhoraram com conduta expectante. Não tivemos nenhum caso de sangramento após a primeira semana.

Observamos nos pacientes submetidos a tonsilectomia maior incidência de disfagia a líquidos no primeiro pós-operatório, de dor e disfagia a sólidos durante a primeira semana e dor durante o primeiro mês, comparado aos grupos submetidos a adenotonsilectomia e adenoidectomia. Esta diferença pode ser atribuída ao fato de que os pacientes submetidos a apenas tonsilectomia apresentam maior média de idade $(22,7$ anos) quando comparados aos outros grupos (A2 = 6,3 anos, $\mathrm{Ad}=9,25$ anos), com processo de cicatrização mais lento e, portanto, recuperação mais demo$\mathrm{rada}^{2}$

No primeiro pós-operatório, dor foi a queixa mais comum (62,5\%), náuseas ocorreram em 22,4\% dos pacientes e vômitos em 19,04\%, freqüência esta parecida com a encontrada em outros estudos ${ }^{7,8,9}$. Febre (temperatura axilar acima de $38^{\circ} \mathrm{C}$ ) foi observada em $6,8 \%$ dos casos neste período, resultado semelhante ao encontrado por Rivas Lacarte ${ }^{9}$. Não encontramos alterações significativas pós-operatórias com relação a febre, náuseas e vômitos entre os grupos A1, A2 e Ad. Otalgia foi significantemente mais freqüente nos pacientes submetidos a A2 no primeiro pós-operatório, sem diferenças em 1 semana e 1 mês.

Em geral os estudos comparam técnicas cirúrgicas a quente (eletrocautério) e a frio (técnicas de dissecção), sendo que a primeira reduz o tempo cirúrgico e o sangramento intra-operatório ${ }^{14}$, porém em geral os pacientes apresentam significantemente mais dor no pós-operatório ${ }^{15,16}$, não sendo procedimento recomendado de rotina ${ }^{15,16}$. Homer et al..$^{17}$ observaram que a técnica de Sluder provoca menos dor no pós-operatório, resultado este não constatado no presente estudo. Não houve diferença entre os grupos com relação à técnica cirúrgica utilizada, também com relação a incidência de disfagia, febre, náuseas, vômitos e sangramento pós-operatório.

\section{CONCLUSÃO}

Consideramos seguro a alta hospitalar dos pacientes submetidos a tonsilectomias após um período de observação clínica de no mínimo 6 horas, prática esta utilizada de rotina em nosso serviço, barateando consideravelmente o custo do procedimento cirúrgico, já que quando ocorre hemorragia ela é muito mais freqüente no pós-operatório imediato5.

As técnicas cirúrgicas de Sluder, dissecção com pinça de Hartmann e com descolador aspirador não apresentam diferença estatística com relação a sangramentos pós-operatórios e outras complicações (náuseas, vômitos, febre, otalgia, dor e disfagia), devendo o cirurgião utilizar a técnica com a qual é mais familiarizado.

\section{REFERÊNCIAS BIBLIOGRÁFICAS}

1. Gabalski EC, Mattucci KF et al. Ambulatory Tonsillectomy and Adenoidectomy. Laryngoscope 1996; 106: 77-80.

2. Randall DA, Hoffer ME. Complications of tonsillectomy and adenoidectomy. Otolaryngol Head Neck Surg 1998; 118(1): 618 .

3. Windfuhr JP, Ulbrich, T. Post-tonsillectomy hemorrhage: Results of a 3-month follow-up. Ear, Nose \& Throat J 2001; 80(11): 790802

4. Feldmann H. 200 year history of tonsillectomy. Images from the history of otorhinolaryngology, highlighted by instruments from the collection of the German Medical History Museum in Ingolstadt]. Laryngorhinootologie 1997; 76(12): 751-60.

5. Windfuhr JP, Chen YS. Post-tonsillectomy and -adenoidectomy hemorrhage in nonselected patients. Ann Otol Rhinol Laryngol 2003 Jan; 112(1): 63-70.

6. Johnson LB, Elluru RG, Myer CM. Complications of adenotonsillectomy. Laryngoscope 2002 Aug; 112(8 Pt 2): 35-6.

7. Castellano P, Gámiz MJ, Bracero F, Santiago F, Soldado L, Solanellas J, Ruiz-Mondéjar A, Esteban F. "Usual" morbidity of pediatric tonsillectomy: a study of 126 cases. Acta Otorrinolaringol Esp 2001 Jun-Jul; 52(5): 390-5.

8. Lee WC, Sharp JF. Complications of paediatric tonsillectomy postdischarge. J Laryngol Otol 1996 Feb; 110(2): 136-40. 
9. Rivas LM. Tonsillectomy as a major outpatient procedure. Prospective 8-year study: indications and complications. Comparison with inpatients. Acta Otorrinolaringol Esp 2000 Apr; 51(3): 221-7.

10. Almeida ER, Campos VAR. Indicações e contra-indicações de adenotonsilectomias. In: Campos CAH, Costa HOO. Tratado de Otorrinolaringologia. Editora Roca; 2002. Cap. 28, 3: 248-52.

11. Rosner B. Fundamentals of Biostatistics. Boston: PWS Publishers. Second edition, 1986.

12. Rakover Y, Almog R, Rosen G. The risk of postoperative haemorrhage in tonsillectomy as an outpatient procedure in children. Int J Pediatr Otorhinolaryngol 1997 Jul 18; 41(1): 29-36.

13. Windfuhr JP, Chen YS. Hemorrhage following pediatric tonsillectomy before puberty. Int J Pediatr Otorhinolaryngol 2001 May; 58(3): 197-204.
14. Raut V, Bhat N, Kinsella J, Toner JG, Sinnathuary AR, Stevenson M. Bipolar seissors versus cold dissection tonsillectomy: a prospective, randomized, multi-unit study. Laryngoscope 2001 Dec; 111(12): 2178-82

15. Carr MM, Muecke CJ, Sohmer B, Naner JG, Finley GA. Comparison of postoperative pain: tonsillectomy by blunt dissection or eletrocautery dissection. J. Otolaryngol $2001 \mathrm{Fev} ; 30$ (1): 10-4.

16. Lassaletta L, Martin G, Villafruela MA, Bolãnos C, Alvarez-Vicent JJ. Pediatric tonsillectomy: post-operative morbidity comparing microsurgical bipolar dissection versus cold sharp dissection. Int. J. Pediatr. Otorhinolaryngol 1997 Sep; 41 (3): 307-17.

17. Homer JJ, Williams BT, Semple P, Swanepoel A, Knight LC. Tonsillectomy by guillotine is less painful than by dissection. Int. J. Pediatr. Otorhinolaryngol 2000 Jan; 52 (1): 25-9. 